\title{
Underwater Communications Using Virtual Time Reversal in a Variable Geometry Channel
}

\author{
A.J. Silva and S.M. Jesus \\ SiPLAB, Universidade do Algarve, PT-8000 Faro, Portugal. \\ \{asilva,sjesus\}@ualg.pt
}

\begin{abstract}
Field experiments using Time-Reversal Mirror(TRM) techniques have shown that the underwater acoustic channel presents a longer stability than it was previously anticipated. Applying such techniques to underwater communications requires that, either the emitted signals are previously filtered by time-reversed replicas of the channel impulse response or that a probe-signal should be transmitted ahead of the data-signal for post channel filtering. In the former case, the time-reversed filtered message is expected to be undone by the actual acoustic channel between the array and the receiver, while in the later, the undoing of the multipath is performed electronically inside the computer and is therefore termed as virtual Time Reversal Mirror(vTRM). The main issues being addressed in recent literature deal with channel stability, focus width and the required array aperture for obtaining reasonable temporal and/or spatial focusing. This paper focus essentially in two practical aspects, generally not addressed: one is to demonstrate the potential application of the vTRM approach to undo the severe intersymbol interference in a real variable geometry channel scenario and, the other, is the importance of optimization of the probe-signal time window length in a real application.
\end{abstract}

Keywords - Coherent underwater acoustic communications, shallow water, time-reversal.

\section{INTRODUCTION}

Ocean exploration requires both remote and in-situ observation systems. Most of those systems have selfrecording capabilities, so that data may be gathered for later off-line analysis. This has been the prevailing philosophy used in the design of autonomous underwater vehicles (AUVs), which are able to perform sophisticated missions with little or no human intervention. Deploying AUVs in more adverse conditions will require tighter coupling with a remote fixed station, so that the unfolding of missions may be assessed and its goals redefined on-line when the need arises. One of the key components that enable the real-time exchange of data between the AUV and the fixed station is a reliable high-speed acoustic communication link.

The underwater acoustic communication channel limitations are mainly due to multipath, that induces severe intersymbol interference in the transmitted waveforms. Attainable reliable data transmission rates are very modest and seldom attain a few kbauds at useful ranges of ten or more water depths. Such rates are often suitable for transmitting telemetry data from untethered measurement instruments, but are clearly insufficient for AUV monitoring. Coherent modulation seems to be the most viable op-

This work was supported under projects INFANTE and INTIMATE of FCT, Lisboa, Portugal and project LOCAPASS(Ministry of Defense - FUP). tion for obtaining higher data rates in the ocean, where the available bandwidth is inherently restricted as a result of frequency-dependent sound absorption. Effective coherent receivers usually exploit spatial diversity and use powerful multichannel equalization algorithms to attain acceptable error rates [1]. A different approach, known as acoustic time-reversal, that fully exploits the spatial coherence of the underwater channel was applied to digital communications in [2], [3], [4], [5], [6].

Acoustic time-reversal was originally proposed by [7], [8] and sucessfully tested at sea in [9]. In this technique, the signal is sent from a source and received in a sufficiently long receiver/source array that, acting like an acoustic mirror reverses the signal and retransmits it back to the source creating a constructive interference at the source location due to medium reciprocity. When applied to digital communications, there is no purpose to send a signal back and forth between source and receiver, instead, assuming that the acoustic channel is sufficiently stable in time, the channel probe at one given time is stored and used to deconvolve the data packets at later times. This process is known as passive phase conjugation [10], [5] and demonstrated with simulated data, in the underwater communication context, in [3]. Since, in this case, time-reversal is done not by the ocean but inside the computer, this technique is also termed as virtual Time Reversal Mirror (vTRM). As its active TRM counterpart, vTRM performance will depend upon the stability of the propagation channel and the ability of the receiving array to correctly sample the most important features of the acoustic field at the useful frequencies. In addition to demonstrating the practical feasibility of TRM in the ocean, the experiment by Kuperman et al., [9] also showed the remarkable temporal stability of this process. Pulses were successfully refocused up to one week after the original recordings. Through simulations Silva et al. [3], showed that the receiving array does not have to span all the water column or be extremely dense, but must intersect most of the energy propagating in the sound channel. Remains an often overlooked problem, which is that of the choice of the time-reversal window length, i.e., the choice of the duration of the signal to be recorded, time-reversed and retransmitted back to the source in the active TRM or, equivalently, the duration of the received probe signal in the case of the vTRM. Typically, the duration of this time window should depend on the time dispersion of the acoustic channel which, in turn, depends on the physical channel properties and the geometry of the experiment. Empirical 


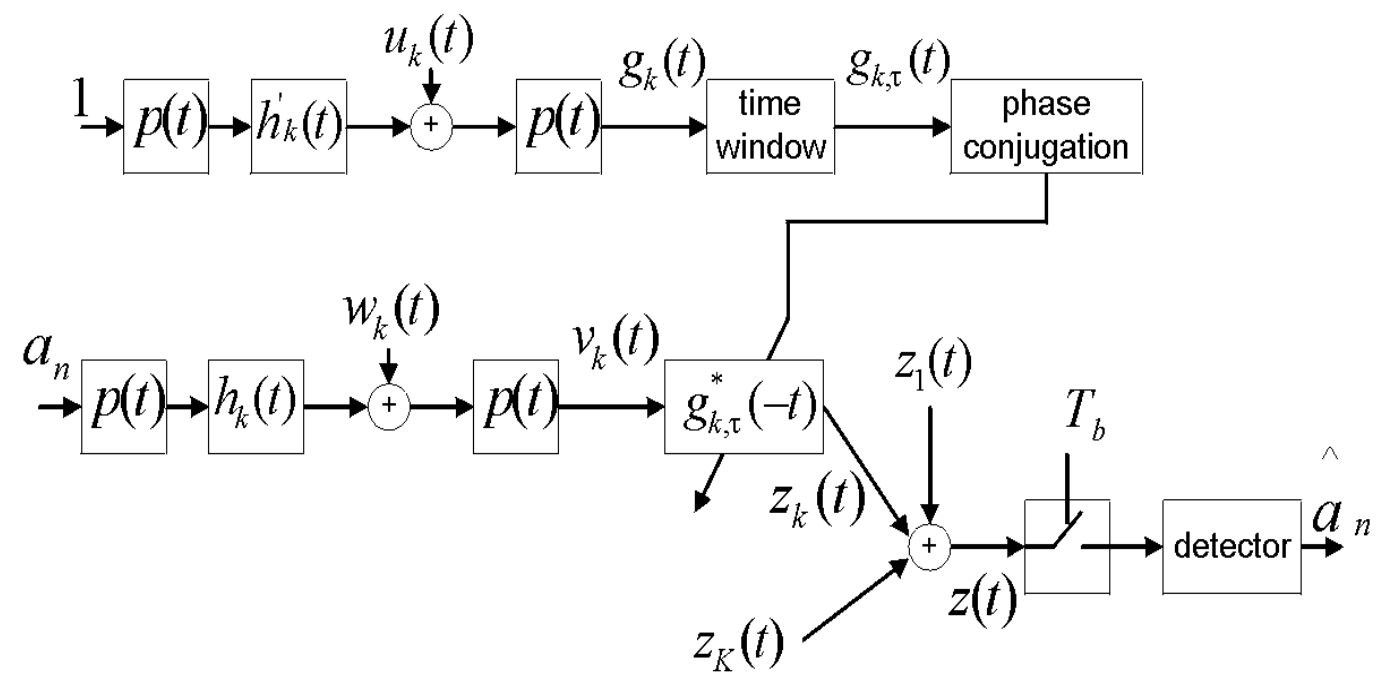

Fig. 1. Block-diagram for the source-channel-receiver with the virtual TRM and for one sensor.

reasoning would suggest that if a short time window fails to include all significant multipath it will result in an imperfect retrofocusing, while a too long time window will reduce the efficiency of the communication system and introduce additional noise in the TRM system. That problem calls for an optimization, that was addressed in [4], [2] though, no convincing theoretical explanations were proposed.

\section{THEORETICAL BACKGROUND}

The objective of this section is to setup the theoretical background to explain the implications of probe signal noise and time window length in vTRM. Let us assume that the transmitted signal is Pulse Amplitude Modulated (PAM) and can be written as

$$
s(t)=\sum_{n=-\infty}^{+\infty} a_{n} p\left(t-n T_{b}\right)
$$

where $a_{n}$ is the symbol sequence, assumed white with power $\sigma_{a}^{2}, T_{b}$ is the symbol period and $p(t)$ is the pulse shape function. Assuming the acoustic channel as a timeinvariant linear system with impulse response $h_{k}(t)$, the received signal at hydrophone $k$ is

$$
r_{k}(t)=h_{k}(t) * s(t)+w_{k}(t)
$$

where $w_{k}(t)$ is an additive zero mean white noise with power $\sigma_{w}^{2}$, assumed to be uncorrelated with the signal and from sensor to sensor. Figure 1 shows the source - channel - receiver representation of the vTRM processing for one single hydrophone. If a probe signal is transmitted the received signal is (path above in figure 1) is written

$$
r_{k}^{\prime}(t)=h_{k}^{\prime}(t) * p(t)+u_{k}(t)
$$

where $u_{k}(t)$ is the channel additive noise sequence with the same properties as $w_{k}(t), h_{k}^{\prime}(t)$ is the same channel impulse response as $h_{k}(t)$ but where the ' denotes that there is an unspecified time delay $t_{0}$ between the two and $p(t)$ is the pulse shape. The receive filter is simply the pulse shape $p(t)$, thus

$$
g_{k}(t)=p^{2}(t) * h_{k}^{\prime}(t)+p(t) * u_{k}(t)
$$

where $p^{m}(t)$ will denote, in this context, the $m$-th self convolution of $p(t)$, i.e.,

$$
p^{m}(t)=p(t) * p(t) * \ldots(\mathrm{m} \text { times }) \ldots * p(t)
$$

where $p^{4}(t)$ is a raised cosine pulse. The time window box just selects a window of length $\tau$, thus

$$
g_{k, \tau}(t)= \begin{cases}g_{k}(t), & t \in[0, \tau] \\ 0, & \text { elsewhere }\end{cases}
$$

Finally, before the vTRM operation is applied to channel $k$, the signal is phase conjugated or, equivalently in the time domain, time-reversed and conjugated. The vTRM output for channel $k$ is therefore

$$
\begin{aligned}
z_{k}(t) & =g_{k, \tau}^{*}(-t) * \nu_{k}(t) \\
& =g_{k, \tau}^{*}(-t) * p(t) * r_{k}(t)
\end{aligned}
$$

where $r_{k}(t)$ is given in equation (2). Replacing (2) and (4) in (8) and summing over the hydrophone index $k$ gives

$$
z(t)=y(t)+x 1(t)+x 2(t)+x 3(t),
$$

where $y(t)$ contains the desired signal information and the other three are noise disturbances, defined as follows

$$
\begin{aligned}
y(t) & =\sum_{n=-\infty}^{+\infty} a_{n} c\left(t-n T_{b}\right) \\
x 1(t) & =\sum_{n=-\infty}^{+\infty} a_{n} e\left(t-n T_{b}\right) \\
x 2(t) & =p^{3}(t) * \sum_{k=1}^{K} h_{k, \tau}^{*}(-t) * w_{k}(t) \\
x 3(t) & =p^{2}(t) * \sum_{k=1}^{K} u_{k, \tau}^{*}(-t) * w_{k}(t) .
\end{aligned}
$$




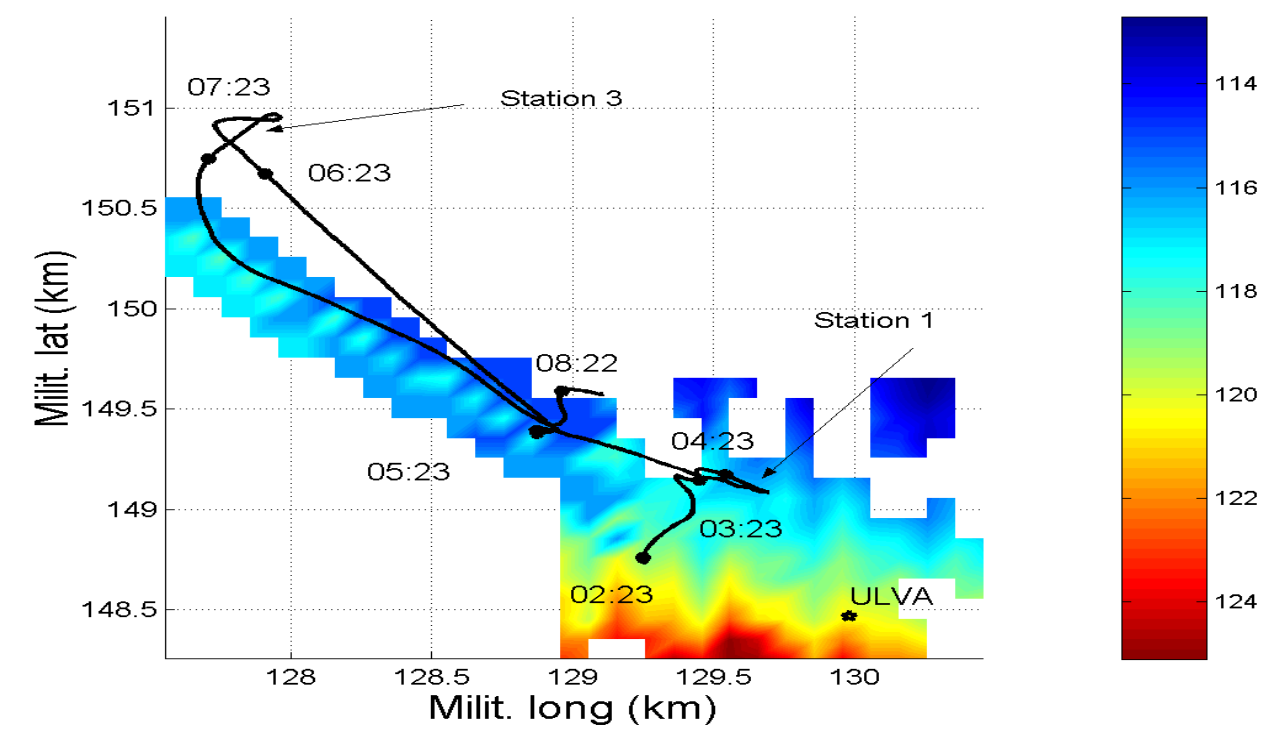

Fig. 2. INTIFANTE'OO Event I source-receiver geometry and bathymetry; ULVA refers to the VLA position.

where

$$
\begin{aligned}
& c(t)=p^{4}(t) * \sum_{k=1}^{K} h_{k}(t) * h_{k, \tau}^{*}(-t) \\
& e(t)=p^{3}(t) * \sum_{k=1}^{K} h_{k}(t) * u_{k, \tau}^{*}(-t) .
\end{aligned}
$$

At this point it is important to characterize each noise disturbance $x 1 \ldots 3$ individually, namely by determining their mean and variance. Their mean is easily calculated since the additive noise is zero mean, then $E[x 1 \ldots 3(t)]=0$. The variance can be obtained as the value of the autocorrelation function at the origin. Since, the noise disturbance $x 3(t)$ results from the convolution of two uncorrelated noise signals $u_{k, \tau}(t)$ (zero mean, non-stationary, time limited) and $w_{k}(t)$ (zero mean, and stationary), its variance will be proportional to the product of the two noise signals variances scaled by the time window length. For $x 2(t)$ it can be seen that its autocorrelation for channel $k$ is

$$
R_{x 2, k}\left(t^{\prime}\right)=R_{p^{3}}\left(t^{\prime}\right) * R_{h, k, \tau}\left(t^{\prime}\right) * \sigma_{w}^{2} \delta\left(t^{\prime}\right),
$$

thus, since the autocorrelation of the sum over the entire array is the sum of the autocorrelations (16) plus the crosscorrelation terms, the autocorrelation of $x_{2}(t)$ is

$$
\begin{aligned}
R_{x 2}\left(t^{\prime}\right)= & \sigma_{w}^{2} \sum_{k=1}^{K} R_{p^{3}}\left(t^{\prime}\right) * R_{h, k, \tau}\left(t^{\prime}\right)+ \\
& +\sum_{i \neq j}^{K} R_{x 2_{i}, x 2_{j}}\left(t^{\prime}\right) .
\end{aligned}
$$

This equation can be further simplified considering that in the first term the summation over hydrophone number applies only to the channel transfer function $h_{k, \tau}$ and that, according to the TRM basic principle [9], for large values of $\tau$ and associated assumptions ${ }^{1}$

$$
\sum_{k=1}^{K} R_{h, k, \tau}\left(t^{\prime}\right) \approx C_{h, \tau}^{\prime} \delta\left(t^{\prime}\right)
$$

thus, finally the $x 2(t)$ variance

$$
\begin{aligned}
\sigma_{x 2}^{2} & =R_{x 2}(0) \\
& =\sigma_{w}^{2} C_{h, \tau}^{\prime} R_{p^{3}}(0)+\sum_{i \neq j}^{K} R_{x 2_{i}, x 2_{j}}(0) .
\end{aligned}
$$

The noise disturbance $x 1(t)$ is treated as a PAM signal with a pulse shape that is a filtered white noise signal similar to the previous disturbance $x 2(t)$. Thus its noise variance can be directly written as

$$
\sigma_{x 1}^{2}=\frac{\sigma_{a}^{2}}{T_{b} 2 \pi}\left[\sigma_{u}^{2} C_{h} R_{p^{3}}(0)+\sum_{i \neq j}^{K} R_{e_{i}, e_{j}}(0)\right]
$$

where, similarly to $x 3(t)$, it was considered that $\tau$ was sufficiently long for the autocorrelation function of $u_{k, \tau}(t)$ to be approximated by a Dirac impulse, thus $\sigma_{u}^{2}=\sigma_{w}^{2}$ and where the constant $C_{h}$ does not depend on the time window interval $\tau$, since on equation (15) the channel impulse response $h_{k}(t)$ is not a function of $\tau$.

Regarding the signal term $y(t)$ in (9) it should be noticed that the TRM basic assumption made in (18) leads to

$$
c(t) \approx p^{4}(t) * C_{h, \tau} \delta(t),
$$

where the constant $C_{h, \tau}$ (and also $C_{h, \tau}^{\prime}$ ) tend to $C_{h}$ when $\tau$ is sufficiently large compared to the duration of the true

${ }^{1}$ i.e., there is a sufficiently large number of hydrophones, the vertical array is spanning the whole water column and the propagation environment is time-invariant. 
channel impulse response. In practice, the most relevan$\mathrm{t}$ factor in ISI limited communication channels is not the amplitude value but the delta function that, by itself, represents a complete ISI elimination. The variances of the noise disturbances $x 1$ and $x 2$ are directly related with the additive noise variance and, at least one of them, also depends of the time window length via the constant $C_{h, \tau}^{\prime}$. The noise disturbance variances also depend on the noise crosscorrelation terms between sensors which were assumed to be negligeable in [2]. An accurate analysis of the noise disturbance variance for any value of the probe signal time window $\tau$, requires the calculation of the autocorrelation function of a time limited white noise sequence, whose closed form expression is not known. Further work is necessary to assess the true influence of those terms on the TRM performance.

(a)

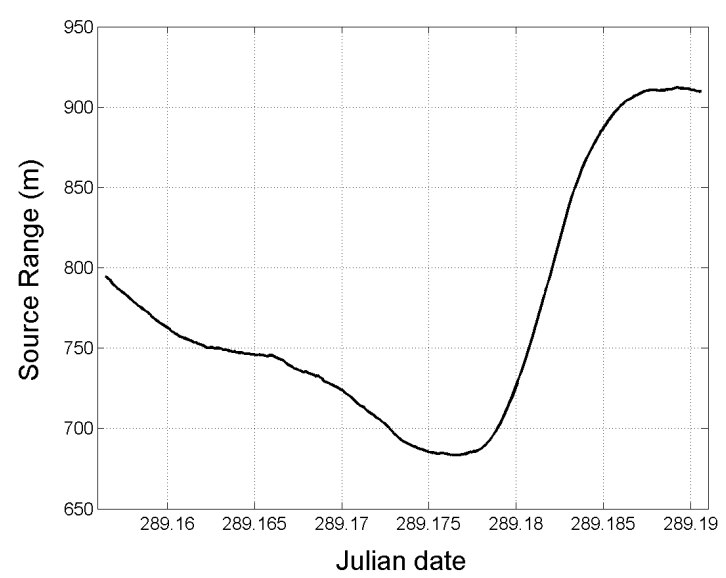

(b)

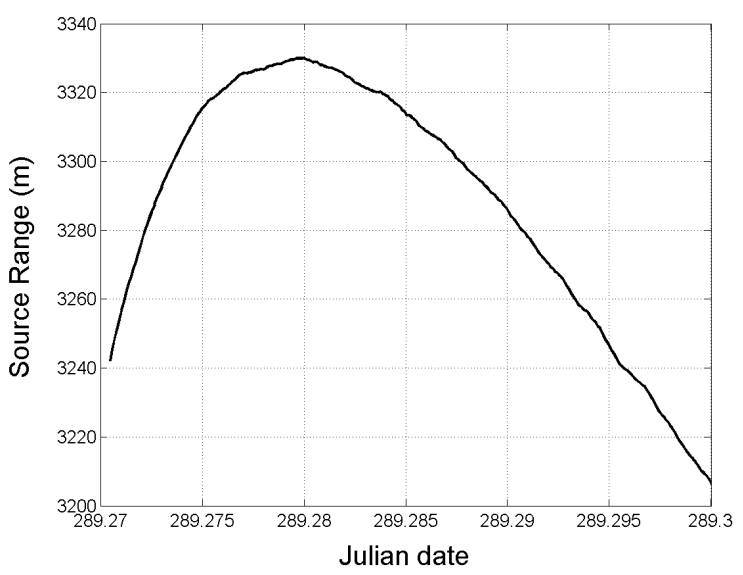

Fig. 3. Source - VLA mooring range measured from GPS data during stations 1 (a) and station 3 (b).

\section{ThE INTIFANTE'00 TIME-REVERSAL EXPERIMENT}

In October 2000 an experiment was conducted in a 120 $m$ depth area of the continental platform off Setúbal, approximately $50 \mathrm{~km}$ south of Lisbon (Portugal). Differential Phase Shift Keying (DPSK) sequences where transmitted from a $70 \mathrm{~m}$ depth acoustic source and received on a 16hydrophone surface suspended vertical line array (VLA) with $60 \mathrm{~m}$ aperture. Due to sound source limitations, the center frequency was chosen equal to $1.6 \mathrm{kHz}$, limiting the bit rates to 75,150 and 300 symbols/sec. Each data packet had a duration of 5 seconds and was preceded by a probe signal with 1 second duration. The source was towed away and then back to the receiving array between approximately $500 \mathrm{~m}$ and $3.3 \mathrm{~km}$ with various stations, as shown in figure 2. While on station the source ship was drifting and, taking into account that the VLA was moving around the mooring in a radius of approximately $100 \mathrm{~m}$, the acoustic link geometry is strongly time variable. That is illustrated by the nominal ship to VLA mooring range, as shown in figure 3, during stations 1 and 3, at approximately 800 and $3200 \mathrm{~m}$ in (a) and (b), respectively.

The acoustic signals received at the VLA were retransmitted via an RF link to onboard the source ship, monitored and stored. The vTRM processing was performed off-line via an inband pre-filter with the pulse shape signal and then baseband shifted. At that point the processing was different for the probe signal and for the actual data packet. For each array channel, the processing was made according to the block diagram shown in figure 1, where (top flow line) the probe signal was timewindowed, phase-conjugated and then applied to the whole data stream (lower flow line). The whole data stream means that it was in fact applied to itself (first second) and then to the data packet (next 5 seconds). Finally, the whole filtered channels are summed over the array, sampled at bit time $T_{b}$ and passed through a symbol detector to obtain the final estimate $\hat{a}_{m}$. As an example, figure 4 shows the $300 \mathrm{bits} / \mathrm{s}$ root-root raised cosine probe signal received at hydrophone 1 (32 $\mathrm{m}$ depth) during station 3 (a) and the resulting array self-vTRM signal output $z(t)$ (b). It can be noted that no acoustic paths can be distinguished in the probe signal of figure 4(a), possibly due to a very tight multipath structure unresolved by the short signal bandwidth and that the vTRM output shows an almost perfect Dirac signal at the focus 4(b). Obviously, this result is optimal in the sense of propagation channel variation and time window length since it represents the self-vTRM output at an optimum window length of 0.15 seconds (see figure 5 and discussion below).

Comparing the estimated vTRM ouput symbols $\hat{a}_{n}$ with the input sequence $a_{n}$ allows for estimating the bit error probability at each trasmission rate and for each station. In order to obtain a statistically valid data sample, three seconds of data where used on each data packet. The results are shown in figure 5 for station 1 (a) and for station 3 (b) as a function of the probe signal time window. The minimum time window duration was chosen to be equal to two times the symbol period $\left(2 T_{b}\right)$.

Several points can be made regarding these results:

a) the error rates are extremely low taking into account the source-receiver distances, the continuous source and array relative moving and the fact that there is no equalization whatsoever, 
(a)

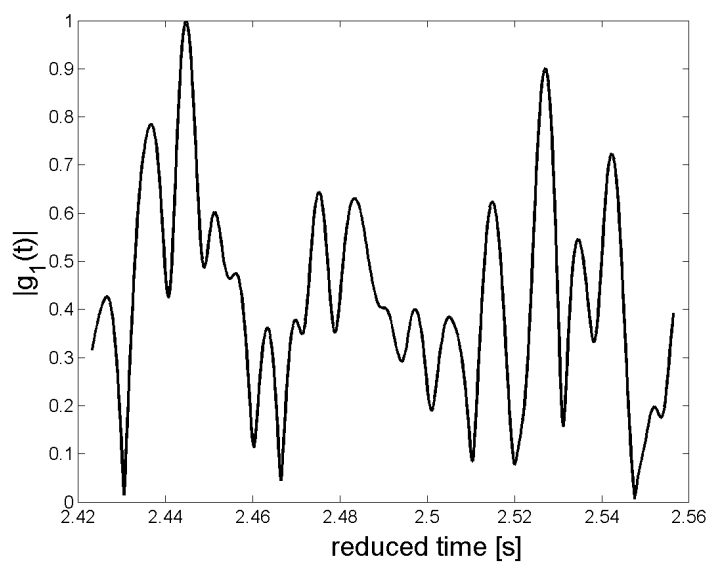

(b)

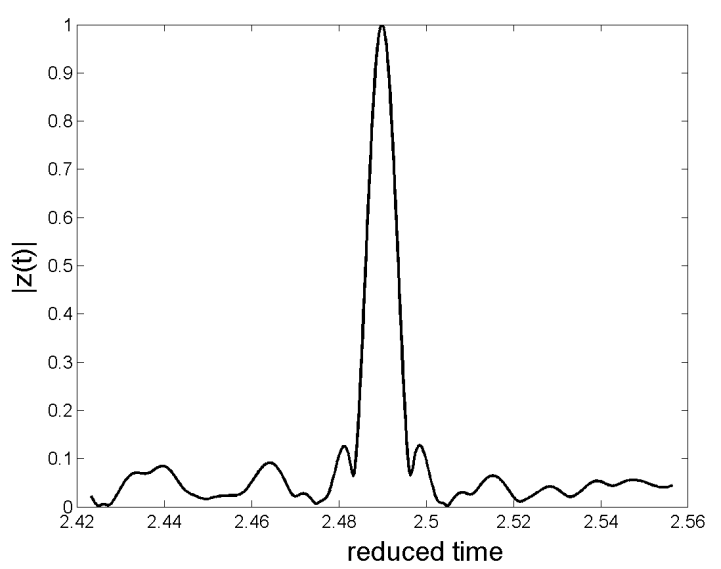

Fig. 4. Self-virtual Time Reversal Mirror with a root-root raised cosine probe signal at 75 bits/s during station 3: received signal on hydrophone 1(a) and vTRM output (b).

b) the error rate at $300 \mathrm{bits} / \mathrm{s}$ is nearly always larger than that at 75 bits $/ \mathrm{s}$,

c) the error rate is larger for station 3 than for station 1 ,

d) for a useful time window variation, the error rate curve always has a minimum. That minimum occours at a shorter time window at the shorter range station, and

e) for some time window durations (over $0.12 \mathrm{~s}$ for station 3 and between 40 and $80 \mathrm{~ms}$ for station 1) at $75 \mathrm{bits} / \mathrm{s}$, no errors were found between the input and output sequences, thus the error rate could not be estimated due to the limited sample size.

\section{CONCLUSION}

This paper explores the possibility of using vTRM as a first stage processing for underwater acoustic communications in shallow water. One of the most crucial steps, in determining vTRM efficiency, is the choice of the probe signal time-window duration. The results obtained on DPSK2 sequences transmitted on a $120 \mathrm{~m}$ depth channel during the INTIFANTE'00 sea trial have shown that vTRM has successfully deconvolved the multipath structure leading to a nearly ISI free output signal. Estimated bit error proba- (a)

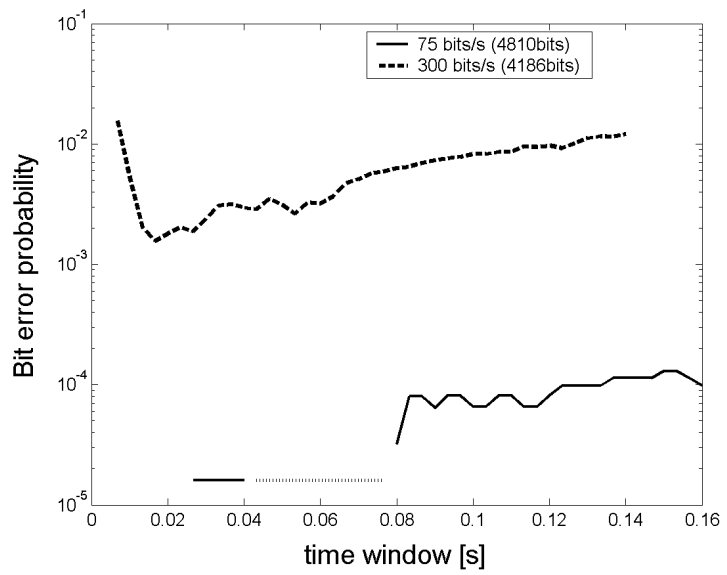

(b)

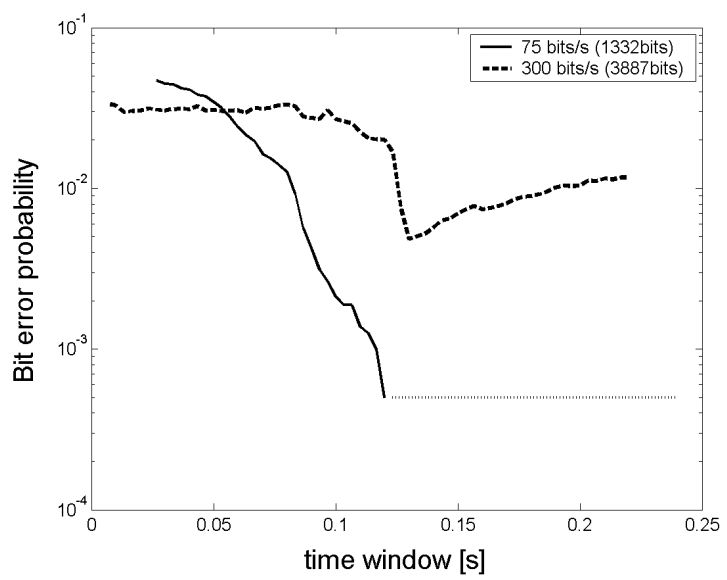

Fig. 5. Empirical probability of error as a function of the probe-signal window duration for 75 bits $/ \mathrm{s}$ (continuous) and $300 \mathrm{bits} / \mathrm{s}$ (dashed): during sation 1 (a) and station 3 (b).

bility was shown to be strongly dependent on the probe signal time window duration. That dependence lead to the appearance of an optimum time window value related with the channel impulse response duration. For this optimum time window duration and for a source-receiver range of approximately $3.3 \mathrm{~km}$, estimated bit error probabilities were of the order of $5 \times 10^{-4}$ and $5 \times 10^{-3}$ at 75 and $300 \mathrm{bits} / \mathrm{s}$, respectively.

\section{ACKNOWLEDGMENT}

The authors would like to thank the SACLANT Undersea Research Centre for the loan of the acoustic sound source, the support of Enrico Muzzi and the NRP D. Carlos I crew during the INTIFANTE'00 sea trial.

\section{REFERENCES}

[1] Stojanovic M., Catipovic J., and Proakis J. Adaptive multichannel combining and equalization for underwater acoustic communications. J. Acoust. Soc. Am., 94(3):1621-1631, 1993.

[2] Gomes J. and Barroso V. A matched field processing approach to underwater acoustic communication. In Proc. of the MTS/IEEE Oceans 1999, pages 991-995, Seattle, USA, 1999.

[3] Silva A., Jesus S., Gomes J., and Barroso V. Underwater acoustic communications using a "virtual" electronic time-reversal mirror approach. In P. Chevret and M.Zakharia, editors, 5th 
European Conference on Underwater Acoustics, pages 531-536, Lyon, France, June, 2000.

[4] Edelmann G.F., Hodgkiss W.S., Kim S., Kuperman W.A., Song. H.C., and Akal T. Underwater acoustic communications using time-reversal. In Proc. of the MTS/IEEE Oceans 2001, pages 2231-2235, Honolulu, Hawai, USA, 5-8 November 2001.

[5] Roussef D., Fox L.J.W., Jackson D., and Jones D.C. Underwater acoustic communications using passive phase conjugation. In Proc. of the MTS/IEEE Oceans 2001, pages 2227-2230, Honolulu, Hawai, USA, 5-8 November 2001.

[6] Jesus S.M. and Silva A. Virtual time reversal in underwater acoustic communications: Results on the intifante'00 sea trial. In Proc. of Forum Acusticum, Sevilla, Spain, September 2002.

[7] Jackson R.D. and Dowling R.D. Phase conjugation in underwater acoustics. J. Acoust. Soc. Am., 89(1):171-181, January 1991.

[8] Dowling R.D. and Jackson R.D. Narrow-band performance of phase-conjugate arrays in dynamic random media. J. Acoust. Soc. Am., 91(6):3257-3277, June 1992.

[9] Kuperman W.A., Hodgkiss W., Chung Song H., Akal T., Ferla C., and Jackson D. Phase conjugation in the ocean: Experimental demonstration of an acoustic time-reversal mirror. J. Acoust. Soc. Am., 103(1):25-40, January 1998.

[10] Dowling R.D. Acoustic pulse compression using passive phaseconjugate processing. J. Acoust. Soc. Am., 95(3):1450-1458, March 1994. 